\title{
IMPACTOS DE LA FRECUENTACIÓN TURÍSTICA EN EL PARQUE NATURAL DE LOS AIGUAMOLLS DE L'EMPORDÀ
}

\author{
Francesc Romagosa Casals \\ Escuela Universitaria de Turismo y Dirección Hotelera \\ Universitat Autònoma de Barcelona
}

\section{RESUMEN}

A lo largo de las últimas décadas el turismo en espacios naturales ha experimentado un proceso de difusión territorial y de aumento del número de practicantes sin precedentes. Uno de los principales retos que tienen la mayoría de espacios naturales protegidos (ENP) es la gestión de la frecuentación turística que en caso de ser masiva o no tener una regulación adecuada puede conllevar una serie de impactos negativos de carácter ambiental y socioeconómico para el espacio visitado. Así, la gestión del uso público se convierte en el elemento clave para el seguimiento y control de los impactos de la frecuentación turística en estos espacios. El artículo analiza el caso de los impactos de la frecuentación en el Parque Natural de los Aiguamolls de l'Empordà, en la Costa Brava. La frecuentación en este parque se concentra principalmente en un área de humedales que está equipada con un itinerario y varios observatorios de fauna y en las distintas playas que forman parte del espacio protegido.

Palabras clave: frecuentación turística, espacios naturales protegidos, Parque Natural de los Aiguamolls de l'Empordà, humedales, playas.

\begin{abstract}
Over recent decades tourism in natural areas has experienced an unprecedented process of territorial diffusion and growth in the number of practitioners. One of the main challenges that the majority of natural protected areas must face is visitor management. If tourist visitation is massified or not properly regulated, it can imply a series of negative environmental and socioeconomic impacts in the area visited. Hence, public use management becomes the key element for the monitoring and control of the impacts that tourist visitation generates in these areas. This article analyzes the case of visitor impacts in the Aiguamolls de l'Empordà Natural Park, situated on the Costa Brava. Tourist visitation in this park is mainly concentrated in a wetland area that is equipped with a trail and some faunal observatories, and at the various beaches located within the protected area.
\end{abstract}


Key words: tourist visitation, natural protected areas, Aiguamolls de l'Empordà Natural Park, wetlands, beaches.

\section{Introducción: el turismo en espacios naturales}

El turismo de naturaleza «es aquél que tiene como principales motivaciones la realización de actividades recreativas y de esparcimiento, la interpretación y/o conocimiento de la naturaleza, con diferente grado de profundidad y la práctica de actividades deportivas de diferente intensidad física y riesgo que usen expresamente los recursos naturales de forma específica, sin degradarlos o agotarlos» (Secretaría General de Turismo, 2004: 14). Por consiguiente, es un tipo de turismo que tiene lugar esencialmente en los espacios naturales, sean protegidos o no, aunque mayoritariamente son los espacios naturales protegidos (ENP) los principales escenarios de esta práctica de ocio. Barrado y Vila (2001) distinguen el turismo de naturaleza del turismo en la naturaleza, en el sentido de que este último además de incluir el turismo de naturaleza también incluye otras actividades turísticas que tienen lugar en espacios naturales pero la motivación principal de las cuales no es necesariamente el contacto con la naturaleza sino la realización de actividades lúdicas y deportivas en un entorno natural, como seria el caso, por ejemplo, del turismo de nieve o del turismo de caza y pesca. En cuanto al ecoturismo, debe ser entendido como una modalidad específica del turismo de naturaleza el objetivo principal de la cual es la visita a espacios naturales bien conservados generando el mínimo impacto ambiental negativo y contribuyendo al desarrollo socioeconómico local.

El turismo de naturaleza y, por extensión, el turismo en espacios naturales, no es un fenómeno reciente. Lo que sí se ha producido durante las últimas décadas es una generalización y expansión de esta tipología de turismo a nivel mundial, convirtiéndose en uno de los segmentos de mayor crecimiento dentro del turismo internacional. Inicialmente era un turismo interno, pero con el tiempo se han ensanchado los destinos que tienen la naturaleza como su principal recurso turístico. Cada vez aparecen destinos más alejados de los grandes centros emisores de turismo, así como una oferta con productos más diversificados y específicos.

En la segunda mitad del siglo XX, la montaña deja de ser el único destino de naturaleza: el turista empieza a descubrir otros espacios naturales también interesantes (zonas húmedas, el mundo submarino...) y destinos tradicionalmente considerados «exóticos» (la sabana africana, la selva amazónica, las latitudes polares...), que basan su éxito en la espectacularidad de sus recursos naturales: paisaje, flora y fauna. En un ritmo similar al que se ha incrementado el número de espacios naturales protegidos a lo largo de las últimas décadas, ha crecido también el número de turistas de naturaleza. En España se estima que actualmente los ENP tienen más de 50 millones de visitantes anuales (Europarc-España, 2006).

Una serie de factores de carácter diverso explicarían esta expansión del turismo en espacios naturales a nivel internacional, entre ellos:

- Los cambios experimentados en la demanda turística durante las últimas décadas, que incluyen: a) una creciente concienciación ambiental y un mayor nivel cultural de los turistas; b) un deseo creciente de estar en contacto con la naturaleza - el denominado «retorno a la naturaleza» por parte de las sociedades urbanas-; y c) un cansancio de los destinos tradicionales maduros de sol y playa, que ha conllevado la búsqueda de destinos alternativos menos masificados y con formas de organización más libres —un turista más activo, aventurero e independiente. 
- Mejora en la accesibilidad hacia los espacios naturales, muchos de los cuales habían sido inaccesibles hasta hace poco tiempo, de forma especial en los países en vías de desarrollo.

- Gran difusión entre los medios de comunicación de masas de recursos turísticos naturales emblemáticos y destinos de referencia que generan interés entre la sociedad para conocerlos y descubrirlos.

La elección de los espacios naturales por parte del turista variará mucho en función de su especialización y de sus intereses. A un mayor conocimiento de la naturaleza, escogerá espacios más valiosos desde el punto de vista ambiental y ecológico, aunque turísticamente no sean muy importantes. En cambio, el turista de naturaleza ocasional y menos informado, se guiará por criterios de espectacularidad (por ejemplo, cataratas, montañas espectaculares u otros fenómenos naturales) y accesibilidad (lugares bien comunicados), o bien por el hecho que pueda realizar un mayor número de actividades, aparte de observar la naturaleza (senderismo, baño, deportes de aventura, etc.) (Barrado y Vila, 2001). Generalmente, son las personas más interesadas en el medio ambiente (los naturalistas, principalmente) los que descubren lugares interesantes, que empiezan a ser visitados y, así, gradualmente, van siendo cada vez más conocidos por el gran público, hasta que finalmente acaban convertidos en destinos importantes del turismo de naturaleza (por ejemplo, los Aiguamolls de l'Empordà o el Delta del Ebro en el caso de Cataluña).

Pero quizás uno de los elementos más importantes que explican la diferencia de uso turístico entre unos espacios y otros, aunque sean muy similares en cuanto a las características de sus recursos naturales, es su nivel de protección: como se ha mencionado anteriormente, los espacios naturales protegidos se han convertido en destinos privilegiados para el turismo de naturaleza. Por este motivo hablamos de un tipo de turismo específico dentro del turismo de naturaleza: el turismo de espacios naturales protegidos. La preferencia por estas áreas protegidas se debe a que son más conocidas por el gran público, se incluyen en las guías turísticas, disponen de información turística y una cierta infraestructura turística (centros de visitantes, itinerarios, etc.). Además, por el sólo hecho de estar protegidas adquieren un cierto prestigio, dado que hay la garantía que sus recursos se conservan y que se preservan especies de flora y fauna en peligro de extinción. Así, atraen tanto a turistas más especializados como a visitantes puntuales, no habituales del turismo de naturaleza. Este hecho, en algunas ocasiones, convierte estos espacios en lugares muy masificados (en España, por ejemplo, el Parque Nacional del Teide - Tenerife - y el Parque Natural del Montseny -Barcelona - son espacios protegidos con más de un millón de visitantes anuales). Esto lleva a la controversia sobre la dificultad de hacer compatible la conservación del espacio natural (aquí debería recordarse que este fue el motivo de su protección y es, de hecho, su función prioritaria) con su uso público, es decir, las visitas de excursionistas y turistas, de forma que se garantice una experiencia de visita de alta calidad (Marion y Reid, 2007).

\section{Los impactos del turismo de naturaleza}

El turismo en general y el turismo de naturaleza en particular se nutren en buena medida de la explotación de recursos naturales y paisajísticos en buen estado de conservación. Pero, paradójicamente, el consumo turístico de estos recursos implica a menudo su degradación o destrucción, hecho que impulsa además la expansión del fenómeno hacia nuevos lugares por explotar. Al mismo tiempo, esta dinámica depredadora degrada la calidad del producto turístico y acaba perjudicando su rentabilidad económica. La actividad turística genera impactos negativos no sólo sobre el medio ambiente sino también sobre la sociedad y la 
economía (Mathieson y Wall, 1982). El Mediterráneo es, sin duda, el mejor exponente mundial de los efectos negativos que el turismo puede tener sobre el medio ambiente y sobre la calidad del propio turismo. Muchas experiencias turísticas iniciadas hace algunas décadas demuestran ahora su inviabilidad ecológica y/o económica o social, su insostenibilidad en definitiva. El reto del turismo del siglo XXI es, pues, la sostenibilidad (EcoMediterrania, 1998; Fullana y Ayuso, 2002; Pérez de las Heras, 2004): el turismo debe romper con su imagen depredadora del medio natural y convertirse, de acuerdo con las nuevas tendencias, en un firme aliado de la conservación, garantizando que el medio ambiente siga aportando al turismo calidad ambiental y recursos turísticos (Figura 1).

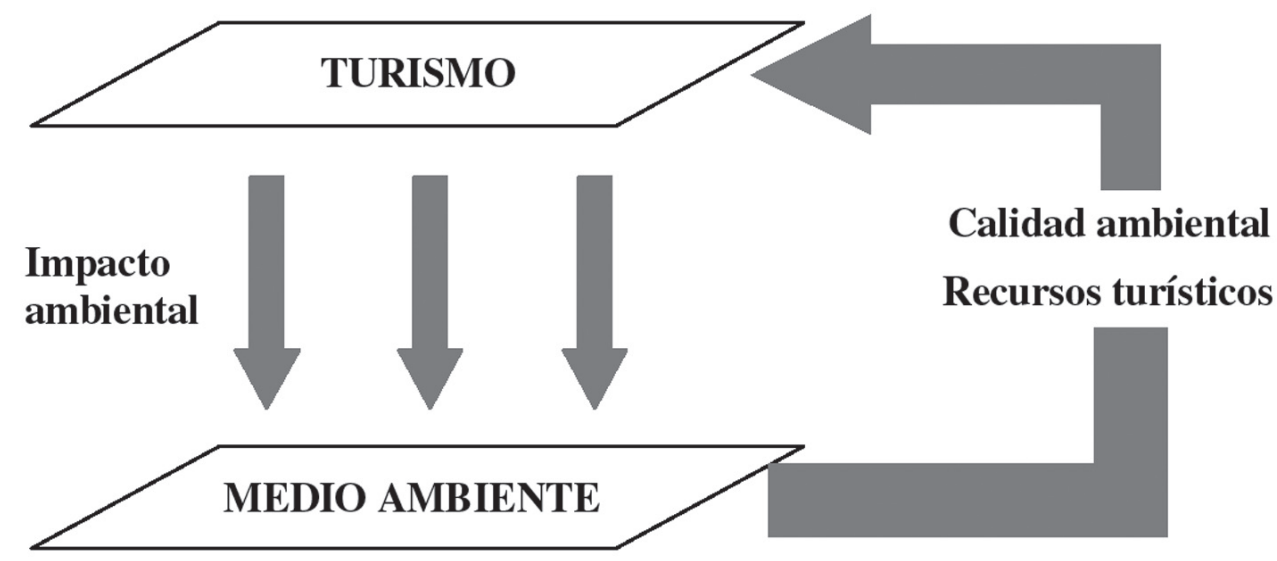

Soporte de la actividad

FIGURA 1. Las relaciones entre turismo y medio ambiente.

Fuente: Campillo et al. (2007).

Desde el punto de vista de la relación entre el turismo en espacios naturales y la conservación del medio ambiente, según Vera (1997) deberán prevalecer las situaciones de indiferencia, es decir, situaciones en las que el turismo no provoca ninguna transformación o esta es inapreciable, o la situación de simbiosis, aquella en que la actividad turística favorece la conservación del medio. Pero lo cierto es que muy a menudo esta relación se manifiesta en forma de conflicto, es decir, aparecen impactos negativos de diferente naturaleza y magnitud. En estos casos, los impactos pueden tener tres orígenes diferentes: a) la construcción de infraestructuras y de equipamientos; b) los efectos de la explotación turística; y c) las actividades llevadas a cabo por los turistas. En este trabajo nosotros nos centramos en el análisis de los dos últimos, que son los que se relacionan más directamente con la frecuentación.

Las actividades de ocio en la naturaleza son menos dañinas en términos globales que otras actividades humanas (agricultura, minería, infraestructuras públicas, etc.). Aun así, teniendo en cuenta que estas actividades se llevan a cabo en áreas bien conservadas y vulnerables, como es el caso de los ENP, los efectos que producen adquieren un valor especial. A menudo se ha remarcado que el turismo de naturaleza puede resultar una contradicción en sí mismo, puesto que la implantación de actividades sobre un medio esencialmente frágil puede conducir a la 
degradación ambiental y, por lo tanto, a la autodestrucción. A pesar de ello, una gestión sostenible de los recursos y las prácticas turísticas ambientalmente respetuosas permiten combinar la protección de la naturaleza, el principal recurso para el desarrollo turístico, con la dinamización socioeconómica. El uso turístico ordenado del medio natural y rural, efectivamente, puede tener un carácter indefinido y es perfectamente compatible con la conservación. En este sentido es también una forma sostenible de explotar los recursos naturales.

La expresión principal del uso turístico del medio natural es la frecuentación. Se entiende por frecuentación la suma de las visitas individuales durante un determinado periodo de tiempo en un ENP o en alguna de las zonas que lo conforman (Europarc-España, 2005). Cuando la frecuentación turística deviene masiva se denomina hiperfrecuentación. En este caso, o cuando no se respetan ciertas normas, el impacto ambiental y socioeconómico puede ser significativo. En realidad, la frecuentación turística es el principal problema ambiental de muchos espacios naturales protegidos. La frecuentación turística sin control perjudica especialmente las especies más sensibles de la fauna silvestre, que necesitan sobre todo tranquilidad (Tapper, 2006). La recolección indiscriminada puede afectar también a determinadas especies de fauna y flora (Hunter y Green, 1995; Eagles et al., 2002). La hiperfrecuentación suele comportar problemas de destrucción de la vegetación por pisoteo excesivo, que a la vez pueden iniciar la aparición de procesos erosivos o, en el caso de las áreas marinas, de degradación de los ecosistemas sumergidos. Sin embargo, el problema más habitual es la proliferación de desechos. La masificación, los desechos y el ruido implican a la vez una pérdida de calidad del paisaje y del producto turístico. Es lo que se denomina banalización (Figura 2).

\begin{tabular}{|c|c|}
\hline $\begin{array}{l}\text { Elementos del } \\
\text { medio }\end{array}$ & Impacto \\
\hline Flora & $\begin{array}{l}\text { - } \text { Pisoteo (pérdida de cubierta vegetal) } \\
\text { - Recolección } \\
\text { - } \quad \text { Cambios en la composiciós florística } \\
\text { - Riesgo por incendios }\end{array}$ \\
\hline Fauna & $\begin{array}{l}\text { - } \text { Estrés } \\
\text { - } \text { Alteración de hábitats y modificación de conductas } \\
\text { - } \text { Intrusión } \\
\text { - } \text { Mecolección indiscriminada, persecución, excesiva caza y pesca } \\
\text { - Riesgo por incendios } \\
\text { - Riesgo por residuos }\end{array}$ \\
\hline Gea & $\begin{array}{ll}\text { - } & \text { Recolección } \\
\text { - } & \text { Erosión y compactación del suelo } \\
\text { - } & \text { Destruminación de las aguas } \\
\end{array}$ \\
\hline Atmósfera & $\begin{array}{l}\text { - Emisión de gases de vehículos } \\
\text { - Contaminación acústica }\end{array}$ \\
\hline Paisaje & $\begin{array}{ll}\text { - } & \text { Banalización (acumulación de personas y vehículos) } \\
\text { - } & \text { Alteración de usos del suelo } \\
\text { - } & \text { Acumulación de residuos } \\
\text { Riesgo de incendios }\end{array}$ \\
\hline
\end{tabular}

FIgURA 2. Impactos ambientales de la frecuentación turística en espacios naturales.

Fuente: Adaptado de Campillo et al. (2007). 


\begin{tabular}{|c|c|c|c|c|c|c|}
\hline \multirow[b]{2}{*}{ Actividades } & \multicolumn{6}{|c|}{ Elementos del medio } \\
\hline & Suelo & Vegetación & Fauna & Agua & Aire & $\begin{array}{l}\text { Medio humano y } \\
\text { paisaje }\end{array}$ \\
\hline $\begin{array}{l}\text { Bicicleta de } \\
\text { montaña }\end{array}$ & $\begin{array}{c}\text { - Procesos erosivos } \\
\text { en puntos concretos } \\
\text { de las rutas }\end{array}$ & $\begin{array}{l}\text { - Pérdida de cubierta } \\
\text { vegetal superficial }\end{array}$ & $\begin{array}{l}\text {-Desplazamientos } \\
\text { de fauna } \\
\text { - Cambios de conducta }\end{array}$ & $\begin{array}{l}\text { - Modificación del } \\
\text { equilibrio fluvial } \\
\text { por el vadeo } \\
\text { continuado }\end{array}$ & $\begin{array}{l}\text { - Aumento sensible } \\
\text { del nivel de ruidos }\end{array}$ & $\begin{array}{l}\text { - Humanización del } \\
\text { paisaje } \\
\text { - Abandono de basuras } \\
\text { - Riesgo de incendios }\end{array}$ \\
\hline Acampada & $\begin{array}{l}\text { - Procesos erosivos } \\
\text { por la concentración } \\
\text { y escavación de } \\
\text { surcos } \\
\text { - Compactación }\end{array}$ & $\begin{array}{c}\text { - Pérdida de cubierta } \\
\text { vegetal superficial por } \\
\text { pisoteo } \\
\text { - Lesiones en árboles } \\
\text { por recogida de leña } \\
\text { - Coleccionismo }\end{array}$ & $\begin{array}{l}\text { - Afección a la } \\
\text { fauna de la zona de } \\
\text { influencia: ruido, } \\
\text { contaminación } \\
\text { - Cambios de conducta } \\
\text { - Proliferación de } \\
\text { especies antropófilas } \\
\text { - Coleccionismo }\end{array}$ & $\begin{array}{l}\text { - Contaminación } \\
\text { por detergentes, } \\
\text { aceites, aguas } \\
\text { fecales, basuras }\end{array}$ & $\begin{array}{l}\text { - Aumento sensible } \\
\text { del nivel de ruidos } \\
\text { en una zona } \\
\text { concentrada del } \\
\text { territorio }\end{array}$ & $\begin{array}{c}\text { - Abandono de basuras } \\
\text { - Riesgo de incendios } \\
\text { - Humanización } \\
\text { del paisaje por } \\
\text { aglomeraciones de } \\
\text { tiendas }\end{array}$ \\
\hline $\begin{array}{l}\text { Senderismo y } \\
\text { montañismo }\end{array}$ & $\begin{array}{l}\text { - Erosión en } \\
\text { caminos } \\
\text { - Cambio de } \\
\text { propiedades y } \\
\text { estructura del suelo }\end{array}$ & $\begin{array}{l}\text { - Pisoteo y aparición } \\
\text { de sendas } \\
\text { - Destrucción de flora } \\
\text { - Coleccionismo }\end{array}$ & $\begin{array}{l}\text { - Permite acceder } \\
\text { a las zonas mejor } \\
\text { conservadas } \\
\text { - Desplazamiento } \\
\text { de fauna }\end{array}$ & $\begin{array}{c}\text { - Contaminación } \\
\text { por filtración de las } \\
\text { fosas sépticas de los } \\
\text { refugios }\end{array}$ & $\begin{array}{c}\text { - Aumento del nivel } \\
\text { de ruidos } \\
\text { - Emisión de humos } \\
\text { por la chimenea } \\
\text { de los refugios y } \\
\text { quema de basuras }\end{array}$ & $\begin{array}{l}\text { - Humanización del } \\
\text { paisaje }\end{array}$ \\
\hline $\begin{array}{l}\text { Escalada en } \\
\text { roca }\end{array}$ & $\begin{array}{l}\text { - Erosión en vías de } \\
\text { acceso y paredes } \\
\text {-Desprendimiento } \\
\text { de rocas }\end{array}$ & $\begin{array}{c}\text { - Afección de la flora } \\
\text { de la base y de la vía } \\
\text { de ascensión }\end{array}$ & $\begin{array}{l}\text { - Afección a } \\
\text { poblaciones de rapaces } \\
\text { - Cambios de conducta }\end{array}$ & & $\begin{array}{l}\text { - Aumento de } \\
\text { ruido por gritos } \\
\text { y utilización } \\
\text { de material } \\
\text { mecanizado }\end{array}$ & $\begin{array}{c}\text { - Abandono de basuras } \\
\text { - Humanización del } \\
\text { paisaje }\end{array}$ \\
\hline $\begin{array}{l}\text { Espeleología y } \\
\text { visita a cuevas }\end{array}$ & $\begin{array}{l}\text { - Expolio de } \\
\text { estalactitas y } \\
\text { estalagmitas }\end{array}$ & $\begin{array}{l}\text { - Alteración de la } \\
\text { diversidad de la flora } \\
\text { por modificación } \\
\text { de las frágiles } \\
\text { condiciones internas }\end{array}$ & $\begin{array}{c}\text { - Alteración de la } \\
\text { diversidad faunística } \\
\text { - Afección a colonias } \\
\text { de murciélagos }\end{array}$ & $\begin{array}{l}\text { - Contaminación de } \\
\text { aguas subterráneas }\end{array}$ & $\begin{array}{l}\text { - Cambios físico- } \\
\text { químicos de la } \\
\text { atmósfera inferior }\end{array}$ & $\begin{array}{c}\text { - Abandono de basuras } \\
\text { - Humanización del } \\
\text { paisaje }\end{array}$ \\
\hline Rutas a caballo & $\begin{array}{c}\text { - Erosión } \\
\text { en caminos, } \\
\text { descansaderos y } \\
\text { abrevaderos }\end{array}$ & $\begin{array}{l}\text { - Descalce de raíces } \\
\text { - Pérdida de cubierta } \\
\text { vegetal superficial }\end{array}$ & - Leves molestias & & & $\begin{array}{l}\text { - Abandono de basuras } \\
\text { - Riesgo de incendios } \\
\text { - Humanización del } \\
\text { paisaje }\end{array}$ \\
\hline $\begin{array}{l}\text { Coches y motos } \\
\text { todo terreno }\end{array}$ & $\begin{array}{c}\text { - Apertura de } \\
\text { nuevos accesos } \\
\text { - Erosión en } \\
\text { pendientes y rampas }\end{array}$ & $\begin{array}{l}\text { - Pérdida de cubierta } \\
\text { vegetal superficial }\end{array}$ & $\begin{array}{c}\text { - Molestia a la fauna } \\
\text { por emisión de ruidos } \\
\text { y gases nocivos } \\
\text { - Atropello } \\
\text { - Fragmentación }\end{array}$ & $\begin{array}{l}\text { - Modificación del } \\
\text { equilibrio fluvial } \\
\text { por vadeo } \\
\text { - Contaminación } \\
\text { por aceites y } \\
\text { carburantes }\end{array}$ & $\begin{array}{l}\text { - Aumento del nivel } \\
\text { de ruidos } \\
\text { - Emisión de gases } \\
\text { nocivos }\end{array}$ & $\begin{array}{c}\text { - Pérdida de calidad } \\
\text { y molestias por } \\
\text { saturación de las } \\
\text { infraestructuras viarias } \\
\text { - Humanización del } \\
\text { paisaje }\end{array}$ \\
\hline $\begin{array}{l}\text { Esquí de } \\
\text { travesía y de } \\
\text { fondo }\end{array}$ & $\begin{array}{l}\text { - Erosión en zonas } \\
\text { con poca nieve }\end{array}$ & $\begin{array}{l}\text { - Seccionamiento de } \\
\text { plantones y arbustos } \\
\text { - Alteraciones de la } \\
\text { floración herbácea }\end{array}$ & $\begin{array}{l}\text { - Afección a fauna } \\
\text { hibernante } \\
\text { - Desplazamiento } \\
\text { de fauna }\end{array}$ & & & $\begin{array}{c}\text { - Humanización del } \\
\text { paisaje }\end{array}$ \\
\hline $\begin{array}{l}\text { Parapente y ala } \\
\text { delta }\end{array}$ & $\begin{array}{c}\text { - Erosión en vías de } \\
\text { acceso }\end{array}$ & $\begin{array}{l}\text { - Destrucción de la } \\
\text { vegetación por la } \\
\text { apertura de pistas de } \\
\text { acceso para vehículos } \\
\text { de apoyo }\end{array}$ & $\begin{array}{l}\text { - Abandono del hábitat } \\
\text { de grandes mamíferos } \\
\text { y aves } \\
\text { - Abandono de nidos } \\
\text { - Efecto rapaz }\end{array}$ & & $\begin{array}{l}\text { - Aumento sensible } \\
\text { del nivel de ruidos } \\
\text { en la zona de } \\
\text { concentración }\end{array}$ & $\begin{array}{c}\text { - Abandono de basuras } \\
\text { - Humanización del } \\
\text { paisaje }\end{array}$ \\
\hline Ultraligero & & & $\begin{array}{l}\text { - Invasión del territorio } \\
\text { de las aves } \\
\text { - Invasiones de nidos } \\
\text { y crías }\end{array}$ & & $\begin{array}{l}\text { - Aumento del nivel } \\
\text { de ruidos }\end{array}$ & $\begin{array}{c}\text { - Molestias a } \\
\text { poblaciones próximas } \\
\text { - Humanización del } \\
\text { paisaje }\end{array}$ \\
\hline $\begin{array}{c}\text { Embarcación a } \\
\text { motor }\end{array}$ & $\begin{array}{l}\text { - Movimientos de } \\
\text { tierra derivados de } \\
\text { la construcción de } \\
\text { embarcaderos }\end{array}$ & $\begin{array}{l}\text { - Acción segadora de } \\
\text { hélices en algas y otras } \\
\text { plantas acuáticas } \\
\text { - Alteración de } \\
\text { procesos fotosintéticos }\end{array}$ & $\begin{array}{c}\text { - Afección a } \\
\text { invertebrados y } \\
\text { branquias de peces y } \\
\text { moluscos por aumento } \\
\text { de turbidez } \\
\text { - Molestias por ruidos } \\
\text { - Afección a aves } \\
\text { acuáticas }\end{array}$ & $\begin{array}{l}\text { - Vertido de aceites, } \\
\text { carburantes y } \\
\text { detergentes } \\
\text { - Aumento de } \\
\text { turbidez }\end{array}$ & $\begin{array}{l}\text { - Aumento del nivel } \\
\text { de ruidos } \\
\text { - Emisión de gases } \\
\text { nocivos }\end{array}$ & $\begin{array}{c}\text { - Molestias a } \\
\text { poblaciones próximas } \\
\text { - Humanización del } \\
\text { paisaje }\end{array}$ \\
\hline
\end{tabular}

FIGURA 3. Impactos potenciales de las actividades deportivas en la naturaleza.

Fuente: Ecotrans (1995). 
Los impactos dependen mucho de las características del visitante y, especialmente, de la actividad practicada y su intensidad (Figura 3). También dependen de la capacidad diferencial del medio de resistir los cambios y, desde luego, de la resistencia y reversibilidad de los ecosistemas. Los impactos pueden llegar a ser inadmisibles cuando suponen un retroceso del estado de conservación del espacio natural o una pérdida de los valores que inicialmente motivaron la declaración como tal.

Pero los impactos negativos causados por el uso turístico del territorio no son sólo ambientales, sino también sociales y económicos. Una frecuentación turística sin control puede interferir negativamente en las actividades agropecuarias y forestales. También varias infraestructuras, como caminos rurales, señales, cabañas, áreas de recreo, etc. pueden sufrir daños o ser objeto de actos vandálicos. Tampoco deben olvidarse los costes sociales y económicos de las emergencias de protección civil (accidentados o extraviados) y los conflictos potenciales entre diferentes usuarios turísticos (por ejemplo, entre senderistas y conductores de motocicletas y/o quads) (Figura 4).

- Molestias a la fauna doméstica

- Daños en campos y cultivos

- Daños en la infraestructura agropecuaria y forestal (vallas, cabañas...)

- Daños en la infraestructura viaria (caminos rurales y forestales)

- Daños en la infraestructura turística (señalización, áreas de recreo)

- Conflictos entre usuarios turísticos (vehículos/peatones)

- Emergencias de protección civil

- Basuras

- Incendios

- Pérdida de calidad de los recursos turísticos

FIGURA 4. Impactos socioeconómicos de la frecuentación turística en espacios naturales. Fuente: Adaptado de Campillo et al. (2007).

Sin embargo, es importante tener presente que la mayor parte de los impactos negativos de la frecuentación se concentran en determinados sectores de los ENP, como los aparcamientos, las áreas de acampada, los observatorios y los caminos, que a menudo sólo representan un porcentaje mínimo de la superficie total del espacio protegido (Newsome et al., 2002). Además, no todos los impactos de la frecuentación turística son negativos. Taylor (2004) menciona algunos de los posibles impactos positivos que el turismo de naturaleza puede provocar sobre el territorio de operación, entre los cuales podemos destacar:

- El creciente apoyo entre el público general hacia los ENP y los aspectos ambientales en general.

- La obtención de beneficios económicos por la población local y la región en general. Esto puede contribuir a que las administraciones tengan más argumentos para impulsar las políticas de conservación de los ENP.

- La obtención de fondos adicionales para el espacio protegido (a través de tasas de entrada, servicios de pago, donativos, impuestos locales, etc.).

- El aumento de interés hacia el patrimonio natural y cultural del territorio.

- El creciente apoyo por parte de la población local hacia los ENP, que a su vez se convierte en un aliado básico para las actividades de los ENP. 
En muchas ocasiones los impactos negativos de la frecuentación turística son producidos principalmente por la ubicación errónea de las actividades recreativas y sus equipamientos, por culpa de una ausencia de planificación previa. Esta carencia de planificación a menudo comporta una infradotación de equipamientos para acoger a los visitantes y/o una carencia de servicios y de oferta de actividades. Lo más habitual es no haber considerado los límites de la capacidad de acogida del espacio y cuando estos son superados, los impactos ambientales y socioeconómicos negativos devienen significativos y se pueden llegar a generar graves problemáticas de gestión. Es por este motivo que la gestión del uso público se convierte en el elemento clave para el seguimiento y control de los impactos de la frecuentación turística en los espacios naturales (Hammit y Cole, 1998; Europarc-España, 2005; Benayas et al., 2007; Boada y Piqueras, 2007). La ordenación de la frecuentación por parte de los gestores de los ENP pasa por ofrecer alternativas de uso en los espacios menos sensibles y por regular estrictamente el acceso a los lugares más frágiles, de forma especial por lo que respecta a la circulación motorizada. Además, de esta manera el producto turístico gana en calidad y, en definitiva, mejora la satisfacción del visitante.

\section{El Parque Natural de los Aiguamolls de l'Empordà (PNAE) y el turismo}

Como estudio de caso del impacto de la frecuentación en un ENP específico se ha escogido uno de los diferentes parques naturales de Cataluña: el Parque Natural de los Aiguamolls (Humedales) de l'Empordà. Este parque natural constituye un ejemplo de espacio natural mediterráneo que sufre la presión de los visitantes en dos ambientes muy frágiles como son las zonas húmedas y los sistemas costeros de playas y dunas litorales.

El Parque Natural de los Aiguamolls de l'Empordà (PNAE), situado en la llanura litoral de la comarca del Alt Empordà (Girona), fue creado en 1983 por parte del Parlamento de Catalunya. El PNAE engloba un territorio formado por espacios naturales (mayoritariamente sectores de marismas, lagunas litorales, estanques de agua dulce interior, playas y dunas) y por espacios agrarios (prados de pasto localmente denominados closes y campos de cultivo). Su entorno, sin embargo, está sometido a una gran presión urbanística y turística, puesto que se encuentra en una de las zonas más frecuentadas y urbanizadas de la Costa Brava: el sector situado entre Roses y L'Escala. La presencia de la marina residencial de Empuriabrava (una de las más grandes del Mediterráneo, con 5000 amarres) hace que el parque esté dividido en dos polígonos, uno al norte y otro al sur de esta urbanización turística (Figura 5).

La superficie protegida como Parque Natural es, desde su creación, de 4.824 ha, de las cuales 849,6 son de Reserva Natural Integral (321,2 ha la Reserva de los Estanys o RNI 1, en el polígono norte, y 522,8 la Reserva de Les Llaunes o RNI 2, y 5,6 la Reserva de la Isla de Caramany o RNI 3, las dos últimas en el polígono sur del parque). Pero con la aprobación de la nueva delimitación del Plan de Espacios de Interés Natural (PEIN) en 2006, la superficie protegida quedó ampliada a 4.973 ha terrestres, más 5.857 ha marinas correspondientes al espacio marino adyacente al parque natural. Todo el espacio está incluido dentro de la Red Natura 2000 de la Unión Europea, así como también fue declarado en 1986 Zona de Especial Protección para las Aves (ZEPA) y, desde 1992, es Zona Húmeda de Importancia Internacional (Convenio de Ramsar). Uno de los principales valores y atractivos del espacio natural es su avifauna, puesto que se han identificado más de 320 especies, entre nidificantes y migratorias. Sus diferentes niveles de protección y el hecho que sea un espacio dónde se puedan observar fácilmente aves han sido elementos clave para que se haya convertido en un destino emblemático para los birdwatchers y los amantes de la naturaleza (Romagosa, 2006). 


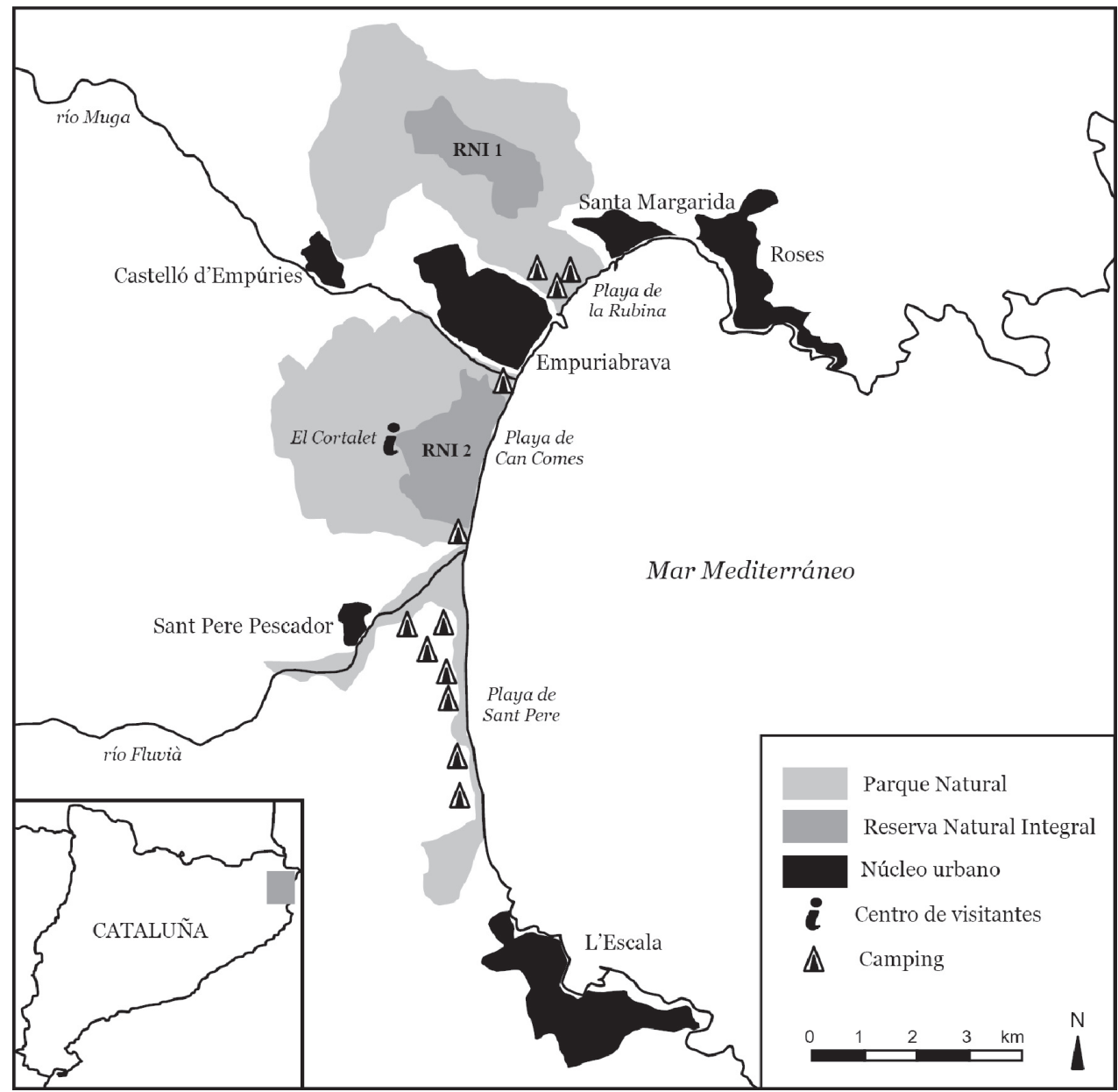

Figura 5. El Parque Natural de los Aiguamolls de l'Empordà.

Elaboración propia.

Teniendo en cuenta que los humedales del Empordà están situados en una zona turística de primer orden, como es la Costa Brava, esto supone unas implicaciones dicotómicas. En primer lugar, el turismo tiene unos efectos desfavorables por la gran presión antrópica que ejerce sobre el medio, tal y como analizaremos a continuación. Pero, por otra parte, el turismo supone una potencialidad positiva para los espacios protegidos. Efectivamente, el interés en desarrollar un producto turístico complementario para la oferta de sol y playa ha sido defendido por la mayoría de agentes implicados en la industria turística. En este sentido, se debe remarcar el elevado grado de complementariedad con la oferta tradicional, que tiene el uso recreativo de los humedales, en tanto que destino de turismo de naturaleza, al mismo tiempo que permite alargar la temporada turística de la zona y combatir la estacionalidad (Romagosa, 2001). A pesar de todo, se constata que en la promoción realizada por los agentes turísticos que operan en la comarca no se da toda la relevancia que se podría dar a los humedales ampurdaneses. 
Como se observa en la Figura 6, la mayor parte de los equipamientos de uso público del parque se concentran en la RNI 2 o en el Polígono sur. Parecería adecuado, por lo tanto, diversificar la oferta para los visitantes, de forma que no se asocie el parque natural sólo con la Reserva Natural de Les Llaunes (RNI 2), como ha sucedido hasta ahora, teniendo en cuenta también el Polígono norte, así como la zona agraria del parque, que es la gran desconocida. En cierto modo, con el objeto de contribuir a la reducción de la hiperfrecuentación de la RNI 2, en 2001 se publicó la guía de itinerarios del parque natural, que incluye diez itinerarios, cuatro de los cuales discurren por el entorno más frecuentado y conocido, pero los otros seis son itinerarios de descubrimiento del resto del espacio protegido (Feliu y Llobet, 2001).

\begin{tabular}{|c|c|c|}
\hline Nombre & Descripción de los equipamientos & Localización \\
\hline $\begin{array}{l}\text { Centro de visitantes } \\
\text { de El Cortalet }\end{array}$ & $\begin{array}{l}\text { Centro de gestión del Parque Natural, con un } \\
\text { punto de atención a los visitantes, un centro de } \\
\text { documentación, un espacio para exposiciones } \\
\text { y un auditorio para conferencias. }\end{array}$ & RNI 2 \\
\hline $\begin{array}{l}\text { Itinerario de Les } \\
\text { Llaunes }\end{array}$ & $\begin{array}{l}\text { Itinerario que se inicia en El Cortalet y } \\
\text { es el más visitado del parque, con trece } \\
\text { observatorios de aves y acceso a la playa. }\end{array}$ & RNI 2 \\
\hline Itinerario de Vilaüt & Itinerario con un observatorio. & RNI 1 \\
\hline $\begin{array}{l}\text { Itinerario del Estany } \\
\text { Europa }\end{array}$ & Itinerario con cuatro observatorios. & Polígono sur \\
\hline Ruta de los Estanys & $\begin{array}{l}\text { Circuito para bicicletas, de } 24 \text { kilómetros, con } \\
\text { un observatorio. }\end{array}$ & $\begin{array}{l}\text { Polígono } \\
\text { norte y } \\
\text { polígono sur }\end{array}$ \\
\hline $\begin{array}{l}\text { Itinerario del Fluvià } \\
\text { y de los campings de } \\
\text { Sant Pere }\end{array}$ & $\begin{array}{l}\text { Circuito para bicicletas que une todos los } \\
\text { campings de Sant Pere Pescador con el } \\
\text { parque. }\end{array}$ & Polígono sur \\
\hline $\begin{array}{l}\text { Itinerario de la } \\
\text { reserva Mig de Dos } \\
\text { Rius }\end{array}$ & $\begin{array}{l}\text { Itinerario por una zona gestionada por la } \\
\text { Obra Social de Caixa Catalunya, con un área } \\
\text { de picnic. }\end{array}$ & Polígono sur \\
\hline $\begin{array}{l}\text { Área de acampada } \\
\text { y servicios del Mas } \\
\text { Matar }\end{array}$ & Área de acampada y servicios. & RNI 2 \\
\hline $\begin{array}{l}\text { Área de acampada } \\
\text { y servicios de El } \\
\text { Cortalet }\end{array}$ & Área de acampada y servicios. & RNI 2 \\
\hline $\begin{array}{l}\text { Área de acampada } \\
\text { de Can Bastons }\end{array}$ & Área de acampada. & RNI 1 \\
\hline
\end{tabular}

FIGURA 6. Equipamientos de uso público del PNAE.

Elaboración propia. 
El parque no dispone de cifras exactas del número de visitantes, si bien según sus estimaciones este número se sitúa alrededor de los 400 visitantes diarios por término medio, con el tope anual durante Semana Santa, cuando acuden masivamente a El Cortalet y sus entornos más de dos mil personas al día. La cifra estimada anual de visitantes es de unos 150.000. A esta cifra, sin embargo, haría falta sumarle otra cifra posiblemente muy superior de personas que también podrían ser catalogadas como usuarios del parque, pero que no entrarían dentro la categoría de visitantes (Hornback y Eagles (1999) y Eagles (2002) se refieren a los usuarios de un ENP como «entrantes» (entrants) en la terminología anglosajona, diferenciándolos de la figura de visitantes). En este caso nos estamos refiriendo a los usuarios de las playas incluidas dentro del PNAE que, en la mayor parte de los casos, no tienen conciencia de encontrarse dentro de un parque natural: simplemente van a la playa a tomar el sol y bañarse o realizar otras actividades lúdicodeportivas. A pesar de ello son usuarios que se encuentran, de hecho, dentro del PNAE y generan unos impactos evidentes sobre el medio.

\section{Los impactos de la frecuentación turística en el PNAE}

Para la realización del análisis y la descripción de los impactos generados por la frecuentación turística en el PNAE se han adoptado técnicas de carácter cualitativo. Por una parte se ha realizado un trabajo de campo consistente en la observación directa (realización de los itinerarios y visita de los diferentes espacios de frecuentación turística del parque en varias ocasiones) y por otra parte se han realizado entrevistas en profundidad con el personal técnico del parque*. La integración de ambas metodologías nos ha permitido contrastar los resultados de las observaciones y las apreciaciones de los entrevistados.

El análisis se ha focalizado en los dos sectores de máxima afluencia del espacio protegido y por consiguiente, dónde aparecen las principales problemáticas derivadas de la frecuentación: la Reserva Natural Integral de Les Llaunes (RNI 2) y las playas del parque natural (Figura 5).

\subsection{La Reserva Natural Integral de Les Llaunes}

\subsubsection{Impacto de los visitantes sobre la Reserva}

En general, el visitante que hace recorridos por los senderos del parque es bastante respetuoso con el entorno. En momentos de máxima frecuentación (especialmente durante la Semana Santa y determinados puentes) se pueden producir problemas de masificación en algunos tramos del itinerario de Les Llaunes y aglomeraciones excesivas en los observatorios. En los momentos de máxima frecuentación, en qué los observatorios están llenos de visitantes, no siempre silenciosos, se constata como la avifauna se siente molesta por esta elevada presencia de personas en sus cercanías, hecho que puede provocar que un grupo de aves huya del entorno del observatorio y se produzca, por lo tanto, una alteración - aunque leve- del hábitat. El impacto puede ser más significativo si esto sucede en época de cría. Los anátidas (patos, ocas y cisnes) acostumbran a ser las especies más sensibles en este sentido.

Se realizaron entrevistas a Josep Espigulé, director del parque; Anna Colomer, responsable de uso público; Jordi Martí, biólogo; y Berto Minovis, responsable del Centro de Fauna, personas a quien agradecemos su colaboración en la investigación. 
La creación, pocos años después de la constitución del parque natural, del estanque artificial de El Cortalet, situado al lado mismo del centro de visitantes del parque ha supuesto un éxito en la gestión de los flujos de visitantes a la reserva natural de Les Llaunes dado que se ha convertido en un auténtico honey pot, consiguiendo que una parte muy significativa de visitantes concentre su visita en el centro de visitantes y el estanque de El Cortalet y sus entornos más inmediatos, rebajando la presión al resto de la reserva.

Por otra parte, el diseño de los itinerarios, con motas de vegetación que en muchos tramos separan el sendero de las zonas de marisma y laguna, así como la ubicación en lugares estratégicos de diferentes observatorios de fauna, permite una correcta interacción entre los visitantes y la fauna de forma que, en general, se produce una coexistencia sin incidencias remarcables entre la fauna y la frecuentación.

Pese a la presencia de carteles recordando la prohibición de tirar basuras, en los momentos de máxima frecuentación es cuando acostumbran a producirse episodios más significativos de presencia de residuos en el suelo. En cuanto a los actos de vandalismo, afortunadamente puntuales, se producen sobre algunos equipamientos de uso público, como por ejemplo en observatorios, rótulos, etc.

Por otra parte, la proliferación de tortugas de Florida (Trachemys scripta) en acequias y estanques de la reserva se debe, con toda probabilidad, según los técnicos del parque, a la introducción que hacen los visitantes, pensándose que este es un ambiente adecuado para esta especie, cuando en realidad es una especie invasora que desplaza las tortugas autóctonas (galápago leproso (Mauremys leprosa) y galápago europeo (Emys orbicularis)). También se han encontrado otras especies exóticas como por ejemplo camaleones, iguanas, pitones, etc. que el Centro de Fauna del parque debe gestionar. En los pequeños espacios húmedos de los alrededores del centro de visitantes el parque ha colocado carteles para concienciar a los visitantes de la necesidad de no introducir animales exóticos en los ecosistemas naturales.

También cabe mencionar la recolección de vegetación por parte de algunos visitantes, desobedeciendo los carteles que indican la prohibición en este sentido. El lirio amarillo (Iris pseudacorus) y el limonium son especies vistosas y atractivas que en la época del florecimiento (primavera) se ven afectadas por su recolección.

Finalmente, la frecuentación en coches a través de la reserva es permitida por el parque durante el verano en un camino que lleva a la playa de Can Comes. Los impactos de esta circulación motorizada en un tramo de la reserva son evidentes en cuanto al estorbo de la fauna, erosión del camino y proliferación de residuos en el parking que da acceso a la playa.

\subsubsection{Impacto de las bicicletas}

La frecuentación de ciclistas (BTT) a lo largo del itinerario que atraviesa la reserva (desde el Centro de visitantes de El Cortalet hasta la laguna de La Massona) provoca en muchas ocasiones una circulación rápida, sin respetar la señalización (velocidad máxima de $10 \mathrm{~km} / \mathrm{h}$ ). La erosión de los caminos es mayor en relación al paso de personas a pie.

Hay un tramo del itinerario dónde la circulación está prohibida a bicicletas y se propone un itinerario alternativo, pero algunos ciclistas pasan por la zona prohibida, sólo apta para peatones porque es estrecha y hay pasos de madera.

En muchas ocasiones se trata de grupos más o menos numerosos (10-15 personas), vestidos con ropa de colores llamativos (fluorescentes y similares), que gritan y hacen ruido, de forma que pueden suponer una molestia para la fauna que se encuentra en los entornos. 
Se han constatado algunos episodios de mortalidad de lagartos (Lacerta $\mathrm{sp}$ ) y otros reptiles y especies vulnerables al pisoteo.

Por otra parte, no son extraños los conflictos entre usuarios (ciclistas versus peatones): el parque recibe quejas de peatones que se han encontrado con molestias en relación a las bicicletas. Con el objetivo de gestionar esta problemática, el parque ha iniciado dos estrategias. Después de haber hecho la prueba de durante un año de dejar circular las bicicletas por el itinerario, se ha visto que propiciaba más elementos negativos que positivos y por lo tanto, como primera estrategia, tiene previsto prohibir nuevamente la circulación de bicicletas e intentar crear un nuevo itinerario alternativo que permita una circulación segregada en relación a los peatones. Pero para hacer este nuevo itinerario el parque debe llegar a acuerdos con los propietarios de los terrenos afectados cosa que de momento no se ha concretado. Una segunda estrategia consiste en potenciar los otros itinerarios ciclistas que ya existen al parque, como es el caso de la Ruta de los Estanys, que pasa por la zona de menos presión en el parque (Reserva de los Estanys) y la nueva Ruta de Sant Pere (sector de Sant Pere Pescador), creada con el apoyo del ayuntamiento de Sant Pere Pescador.

\subsubsection{Impacto de los campings}

Dentro de la superficie protegida del PNAE hay cinco campings, todos ellos situados en la franja costera, junto a las playas. Tres de ellos se encuentran en el sector de La Rubina y los otros dos en el entorno de la reserva de Les Llaunes (uno al norte y el otro al sur). Aunque se trata de una actividad más bien próxima al turismo de masas de sol y playa y, por lo tanto, poco vinculada al turismo de naturaleza, están incluidos dentro de la superficie protegida por el hecho que en el momento de la creación del parque natural ya existían en la zona y no fueron expropiados y actualmente están integrados al parque natural a través de un Plan Especial de Zonas de Acampada.

Por su parte, en el término municipal de Sant Pere Pescador hay siete campings, cinco de los cuales aunque no están situados dentro de la superficie del PNAE, sí que se encuentran adyacentes al espacio protegido (en la zona de la playa de Sant Pere). Se dio el caso de un camping de Sant Pere Pescador que estaba invadiendo espacio del PNAE y en 2007 se restauró la superficie que ocupaba de forma ilegal.

En general son campings que sólo tienen una actividad estacional, durante el verano, pero que implican la presencia masiva de usuarios sobre los ecosistemas naturales (tanto en las zonas de humedales como, especialmente, en las playas y los sistemas dunares) (véase el punto 4.2), provocando efectos perjudiciales sobre los ecosistemas, como por ejemplo la contaminación acústica y la contaminación lumínica.

Otro impacto indirecto de la presencia de los campings al lado mismo del espacio natural es la presencia de gatos y perros que, al acabar la temporada de verano, salen de los campings y actúan como depredadores sobre determinadas especies animales.

\subsubsection{Impacto de la acampada}

La acampada en el parque está prohibida (obviamente, fuera de los campings regulados por el Plan Especial de Zonas de Acampada previamente citados), excepto durante dos meses del verano, cuando se realizan los Campos de Trabajo y los Campamentos de Naturaleza, en dos sectores habilitados a tal fin (Área de acampada de El Cortalet y Área de acampada del Mas Matar).

Para reducir el impacto de la acampada se ha regulado a la baja el número de participantes ( 24 en cada turno). En general se es muy estricto en relación al control de 
los impactos potenciales (impacto acústico de los grupos, de gestión de residuos, etc.) y no se detectan impactos negativos significativos sobre el entorno asociados a estas actividades.

\subsubsection{Impacto de las carreteras}

Aunque no se encuentran dentro de la reserva natural de Les Llaunes, la presencia de carreteras que atraviesan el PNAE (especialmente las carreteras de Figueres a Roses y de Castelló d'Empúries a Sant Pere Pescador), con una intensidad de tránsito muy elevada, implican a menudo mortalidad de animales que cruzan las carreteras (especialmente nutrias (Lutra lutra), erizos (Erinaceus sp), turones (Mustela putorius), jabalíes (Sus scrofa), sapos comunes (Bufo bufo), etc.), pese a los pasos de fauna diseñados para evitar estos accidentes.

\subsection{Las playas del parque natural}

\subsubsection{Impacto de los usuarios de las playas}

La presencia abundante de personas en las playas del PNAE, especialmente durante el verano, provoca la proliferación de desechos en las mismas playas y sus entornos. Al tratarse de playas naturales y/o seminaturales (poco antropizadas) en muchos tramos no disponen de papeleras o contenedores de residuos y, pese a la presencia de carteles pidiendo a los usuarios de la playa que se lleven los desechos, en muchas ocasiones el parque debe proceder a la limpieza de la playa. En otras ocasiones no se puede responsabilizar a los usuarios de la presencia de residuos a las playas, puesto que son residuos que ha traído el mar en momentos de temporales marinos (las denominadas llevantades) y, por lo tanto, tienen un origen más lejano.

\subsubsection{Impacto de la circulación de vehículos y personas en las dunas}

El paso de personas a través de las dunas y de los sectores de trasplaya con vegetación y fauna propias de estos ambientes tan frágiles y sensibles provoca la erosión de las dunas, con la creación de nuevos caminos entre las dunas y la consiguiente destrucción de la vegetación dunar, como es el caso del barrón (Ammophila arenaria), la oruga marítima (Cakile maritima), la barrilla (Salsola kali), el nardo marítimo (Pancratium maritimum), etc. En los sectores dónde es muy frecuente el paso de perros (por ejemplo, en La Rubina) debe sumarse el impacto de la nitrificación del suelo por la presencia de excrementos.

En cuanto a la fauna, es especialmente remarcable la afectación del hábitat del chorlitejo patinegro (Charadrius alexandrinus) por pisoteo de los nidos por parte de los usuarios o por la acción de los perros, que se comen los huevos de esta ave que nidifica en la playa. Entre 1997 y 2007 la presencia de esta especie en las playas del PNAE se ha visto reducida a la mitad, siguiendo la tendencia detectada también en otras muchas playas europeas (Martí, com. pers.). El ostrero (Haematopus ostralegus) y el charrancito común (Sterna albifrons) eran otras especies que antiguamente habían criado en las playas del parque pero ya hace tiempo dejaron de hacerlo. Otras aves, como el playero rojizo (Calidris canutus) o el zarapito real (Numenius arquata) también descansan en la playa todo el año, pero los usuarios de la playa tienden a ahuyentarlos. Pero no sólo las aves sufren los efectos de la presencia humana en playas y dunas: la lagartija colilarga (Psammodromus algirus), 
por ejemplo, también ha visto reducir notablemente su presencia en estos ambientes en la medida que ha ido aumentando la frecuentación humana.

Este impacto es todavía mucho más intenso cuando hablamos de la circulación de coches y, especialmente de vehículos todo terreno y quads en sectores de playa y sistemas dunares. La erosión y la transformación del hábitat provocada por estos vehículos —que tienen prohibido el acceso a estos espacios_ es mucho más significativa que la que provoca el pisoteo de las personas.

Las estrategias de gestión que ha adoptado el parque para hacer frente a estas problemáticas son esencialmente dos. Por una parte, la delimitación del perímetro de las zonas de dunas más sensibles mediante estacas, para evitar tanto el paso de vehículos como de personas (Figura 7). Es una estrategia de gestión que ya se ha aplicado en las playas de La Rubina y algunos sectores de la playa de Sant Pere. Se han creado zonas de aparcamiento para vehículos. El caso de los quads, sin embargo, es preocupante porqué en estos casos dónde las dunas han sido protegidas con estacas, en alguna ocasión estos vehículos han roto las estacas y atravesado las dunas. Por otra parte, el cierre de la playa es otra estrategia de gestión que se aplica a la playa de Can Comes (RNI 2) cada año entre el 1 de abril y el 15 de junio, durante la época de nidificación del chorlitejo patinegro y otras aves de playa. La playa queda cerrada a la circulación de personas.

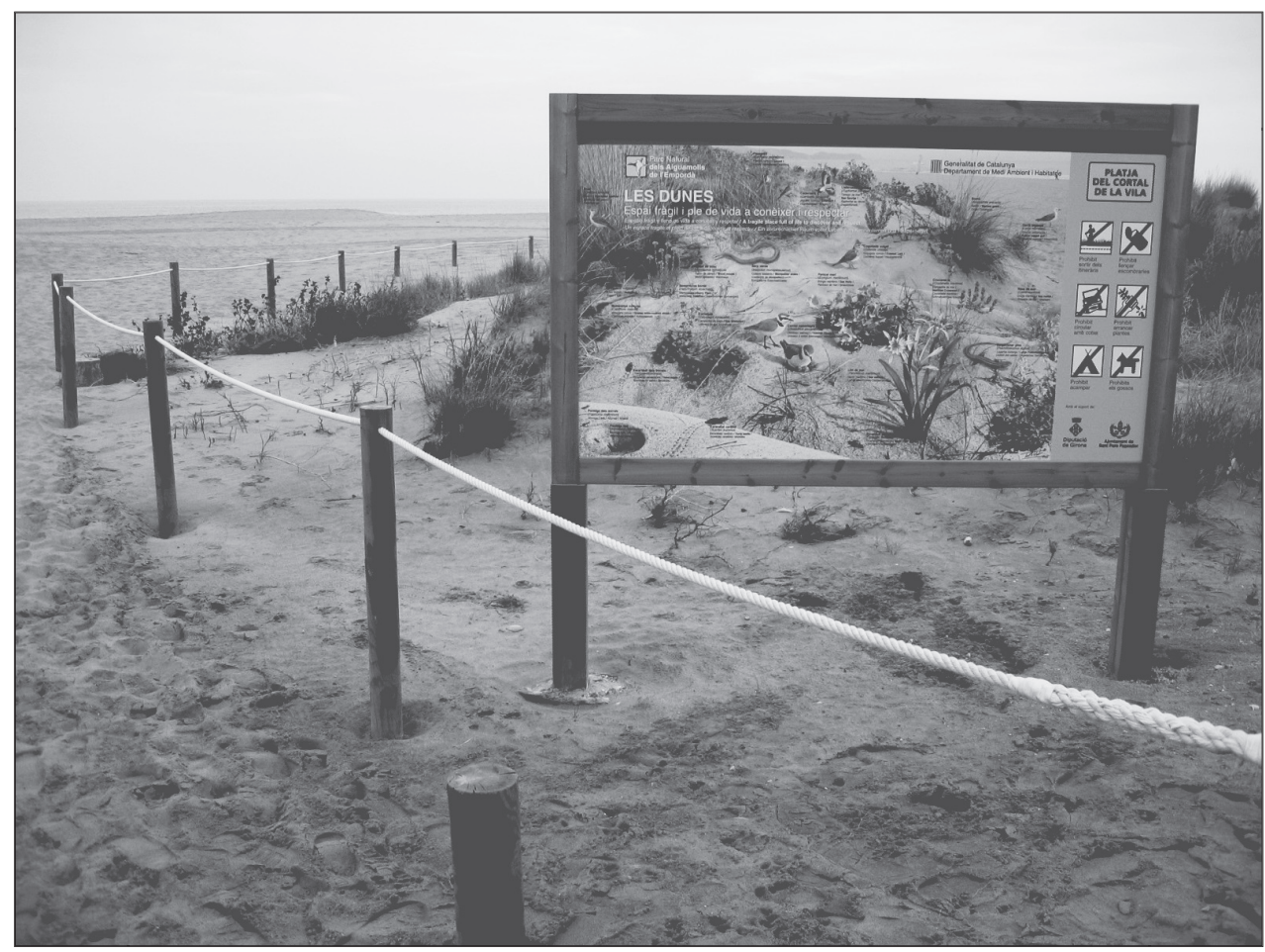

Figura 7. Delimitación del perímetro de las dunas de la playa de Sant Pere y plafón interpretativo. 


\subsubsection{Impacto de las embarcaciones}

Las marinas de Empuriabrava (Castelló d'Empúries) y de Santa Margarida (Roses) suman más de 6.000 amarres de embarcaciones deportivas. Ambas marinas se encuentran en el entorno de las playas del PNAE. Esto genera una fuerte presión para las playas, sobre todo en época estival, cuando muchas embarcaciones quieren acceder a ellas, y de forma particular la playa de Can Comes en el periodo en qué se encuentra cerrada al público. No obstante, recibe mucha más presión el vecino Parque Natural del Cabo de Creus que, al tener pequeñas calas y un paisaje más abrupto, atrae más la atención de los usuarios de las embarcaciones de este sector de la Costa Brava.

En la desembocadura del río Fluvià debe procederse periódicamente a su dragado para que las embarcaciones del Club Náutico de Sant Pere Pescador (situado en el río Fluvià) puedan tener acceso al mar. Los impactos de este dragado, sin embargo, no han sido evaluados.

En cuanto a la afectación sobre el medio marino por parte de las embarcaciones, es la misma que en otros lugares, siendo los principales impactos el vertido de hidrocarburos y aceites, el vertido de residuos sólidos, el vertido de aguas residuales sin depurar, el impacto de las anclas sobre el fondo marino, entre otros.

\subsubsection{Impacto del kite surf}

La práctica del kitesurfing se ha extendido notablemente en las playas del PNAE a lo largo de los últimos años, especialmente en la playa de Sant Pere Pescador, al ser una zona con mucho viento, elemento indispensable para esta práctica deportiva marítima. Con el objeto de regular estas prácticas, la Dirección General de Costas delimitó unas zonas de las playas donde estaba permitida la actividad (esencialmente unos sectores de la playas de La Rubina y de Sant Pere). En la playa de Can Comes (RNI 2) está prohibida, aunque eventualmente se encuentran practicantes ilegales.

Los principales impactos del kite surf se producen en el momento de preparar el material sobre las dunas, afectando la vegetación dunar y las aves nidificantes de playa por pisoteo. Es probable que las velas del kite surf en movimiento también afecten los aláudidos (aves que marcan territorio suspendidos en el aire), particularmente las alondras (Alauda arvensis).

También causan molestias a los demás usuarios de las playas, tanto en el momento de preparación sobre la playa como cuando realizan la actividad ya dentro del mar, constatándose la incompatibilidad con la práctica de los baños de sol y de mar.

\section{Conclusiones y retos de futuro}

Con este trabajo hemos hecho una primera aproximación a la problemática de la frecuentación turística en espacios naturales, con un estudio más detallado sobre unos espacios naturales muy sensibles como son los humedales y los sistemas dunares y de playa que encontramos en el Parque Natural de los Aiguamolls de l'Empordà. Los impactos detectados son propios de espacios naturales que tienen una frecuentación de visitantes relativamente intensa, especialmente en determinados equipamientos como son itinerarios con observatorios de fauna o bien en playas turísticas con sistemas dunares de elevado interés ecológico. Aunque el listado de impactos es relativamente extenso y diverso, la mayoría de impactos no pueden ser catalogados como significativos. Los impactos más relevantes son los que se localizan fuera de las reservas naturales, especialmente en el sector 
de playa y dunas litorales, sometidos a una fuerte presión de visitantes (y, recordemos, de usuarios) y actividades diversas, muchas de ellas no compatibles con la conservación de los ecosistemas naturales.

En cualquier caso, hace falta una mayor profundización en el conocimiento de la frecuentación turística en el parque, realizando nuevos estudios que aporten datos cuantitativos en número exacto de visitantes por periodos temporales (a lo largo del día, a lo largo de la semana, a lo largo del año), que permitan conocer bien la intensidad de uso del espacio, así como datos que permitan conocer la distribución de estos visitantes, sus demandas y preferencias. Tal y como explica Eagles (2002: 5):

«knowledge of public-use activity, location and volume is needed to evaluate, protect and enhance viable natural ecosystems, including endangered and threatened plant and animal species. Hence, visitor-use data (such as visitation rates, level of pressure on sensitive areas, known peak loads at nearby areas, and records of resource erosion at certain volumes) is essential when managing wildlife and their habitats, to ensure resource use remains within acceptable limits (e.g. damage should be minimal and reparable)».

Por otra parte, es preciso que los gestores del espacio protegido desarrollen nuevos instrumentos de seguimiento y control de la frecuentación, así como una regulación de usos bajo la forma, por ejemplo, de un Plan de Uso Público del parque, como los que ya tienen algunos ENP españoles desde hace tiempo (Such, 2003; Europarc-España, 2005), más que basarse en las estrategias tradicionales de la gestión del día a día o del ensayo y error. Un Plan de Uso Público incluye estudios específicos que permiten caracterizar la demanda y proponer posteriormente diversos programas de interpretación y educación ambiental que ayuden a regular el uso público con múltiples finalidades. En este sentido, debe remarcarse la importancia de los programas educativos para hacer frente de manera efectiva a los impactos negativos de la frecuentación, tal y como ya se ha puesto de relieve en varios trabajos (mencionados por Marion y Reid, 2007).

El reto de los gestores de los ENP consiste en encontrar un equilibrio entre las demandas de los usuarios (la satisfacción de la experiencia del visitante, en definitiva) sin que estos provoquen pérdidas irreversibles en los recursos naturales. El objetivo último debe ser, evidentemente, la reducción de los impactos negativos de la frecuentación, buscando el mantenimiento de un equilibrio entre la conservación de los recursos y su explotación. Una frecuentación relativamente elevada si va acompañada de una correcta estrategia de gestión puede suponer unos impactos negativos mínimos sobre el entorno.

Las estrategias de gestión de la frecuentación pueden ser muy diversas (Hammit y Cole, 1998; Newsome et al., 2002). Un tipo de estrategias son de carácter indirecto e incluyen acciones como por ejemplo: a) planificar correctamente la localización de los equipamientos de uso público (cosa que permite gestionar y conducir los flujos de visitantes); b) la propia gestión de los equipamientos (vigilancia, mantenimiento, etc.); c) la restauración y conservación de zonas degradadas; d) acciones de información y concienciación de los visitantes; e) el establecimiento de tasas de entrada en el ENP. Algunas de estas estrategias, dirigidas a la conducción de visitantes, pueden estar diseñadas para favorecer la concentración de visitantes en determinadas zonas (ofreciendo áreas de recreo y promoviendo el uso y la conservación de los caminos rurales tradicionales, evitando así la presión y reduciendo los impactos negativos sobre las zonas más sensibles) o, por el contrario, para favorecer la dispersión de visitantes (este último caso se suele aplicar en ENP con bajos niveles de frecuentación). 
Otro grupo de estrategias, en este caso más directamente dirigidas a la regulación de los visitantes, incluyen, entre otras: a) el establecimiento de unos límites de visitantes en determinadas zonas (número global, medida de los grupos, tiempos de estancia); b) la regulación - mediante la zonificación- del acceso de visitantes a determinadas zonas (que pueden incluir el cierre temporal o permanente, así como la prohibición de acceso a determinados tipos de visitantes en función, por ejemplo, del vehículo utilizado); c) la vigilancia y el control en el cumplimiento de la normativa de uso público del ENP.

En cada caso deberán valorarse cuáles son las mejores estrategias que deben implantarse a partir de la experiencia adquirida y de las prioridades de la gestión del espacio protegido. En cualquier caso es necesario establecer programas de seguimiento que permitan evaluar constantemente el impacto de la frecuentación así como los resultados de las diferentes actuaciones de gestión llevadas a cabo. También debe tenerse muy presente que en todos los casos es preciso incidir tanto sobre los visitantes como sobre los usuarios del espacio protegido. Y, finalmente, no debería hacer falta decir que es básico disponer de suficientes medios humanos, técnicos y económicos que permitan garantizar una correcta gestión de la frecuentación, condición que en muchos ENP, como es el caso del PNAE, desgraciadamente no se cumple.

\section{Bibliografía}

BARRADO, D.A.; VILA, M. (2001). «Turismo en espacios de montaña y naturales», en BARRADO, D.A.; CALABUIG, J. (eds.) Geografía mundial del turismo. Editorial Síntesis. Madrid, pp. 123-149.

BENAYAS, J.; GARCÍA, D.; MUÑOZ, M.; TEJEDO, P. (2007): «Perspectivas actuales y retos futuros en la gestión de las actividades de uso público en la naturaleza», en BOADA, M.; BENAYAS, J. (coords.) Naturaleza y uso público: movilidad, impactos y propuestas. Fundación Abertis. Barcelona, pp. 37-48.

BOADA, M.; PIQUERAS, S. (2007): «Naturaleza y uso público: marco conceptual, movilidad e impactos sobre el patrimonio natural», en BOADA, M.; BENAYAS, J. (coords.) Naturaleza y uso público: movilidad, impactos y propuestas. Fundación Abertis. Barcelona, pp. 11-34.

CAMPILLO, X.; LLURDÉS, J.C.; ROMAGOSA, F. (2007): «El impacto ambiental y sociocultural del turismo», en PRIESTLEY, G.K.; LLURDÉS, J.C. (eds.) Estrategia y gestión del turismo en el municipio. Servei de Publicacions de la Universitat Autònoma de Barcelona. Bellaterra, pp. 57-72.

EAGLES, P.F.J. (2002): «Tourism-use measurement and reporting in parks and protected areas», en Parks, $\mathrm{n}^{\mathrm{o}} 12$ (1), pp. 3-10.

EAGLES, P.F.J.; MCCOOL, S.F.; HAYNES, C.D.A. (2002): Sustainable tourism in protected areas: Guidelines for planning and management. UICN. Gland.

ECOMEDITERRANIA (1998): Turismo sostenible en el Mediterráneo. Guía para la gestión local. Brau Edicions. Girona.

ECOTRANS (1995): Actividades recreativas en la naturaleza. Manuales para la mejora de la calidad ambiental. Vol. 3. Secretaría General de Turismo. Madrid.

EUROPARC-ESPAÑA (2005): Manual sobre conceptos de uso público en los espacios naturales protegidos. Fundación Fernando González Bernáldez. Madrid.

EUROPARC-ESPAÑA (2006): Anuario Europarc-España del estado de los espacios naturales protegidos 2005. Fundación Fernando González Bernáldez. Madrid.

FELIU, P.; LLOBET, T. (2001): Parc Natural dels Aiguamolls de l'Empordà. Guia d'itineraris. Brau Edicions. Girona.

FULlANA, P.; AYUSO, S. (2002): Turismo sostenible. Rubes Ed. y Generalitat de Catalunya. Barcelona.

HAMMIT, W.E.; COLE, D.N. (1998): Wildland recreation. Ecology and management (2a Ed.). John Wiley \& Sons. New York. 
HORNBACK, K.E.; EAGLES, P.F.J. (1999): Guidelines for public use measurement and reporting at parks and protected areas. UICN. Gland.

HUNTER, C.; GREEN, H. (1995): Tourism and the environment. A sustainable relationship? Routledge. Londres.

MARION, J.L.; REID, S.E. (2007): «Minimising visitor impacts to protected areas: The efficacy of low impact education programmes», en Journal of Sustainable Tourism, $\mathrm{n}^{\circ}$ 15(1), pp. 5-27.

MATHIESON, A.; WALL, G. (1982): Tourism. Economic, physical and social impacts. Longman Scientific Technical. Londres.

NEWSOME, D.; MOORE, S.A.; DOWLING, R.K. (2002): Natural area tourism. Ecology, impacts and management. Channel View Publications. Clevedon.

PÉREZ DE LAS HERAS, M. (2004): Manual del turismo sostenible. Ed. Mundi-Prensa. Madrid.

ROMAGOSA, F. (2001): «Els aiguamolls de l'Empordà: de la destrucció a la protecció i l'ús turístic», en Estudis de Turisme de Catalunya, $\mathrm{n}^{\mathbf{0}}$ 9, pp. 11-15.

ROMAGOSA, F. (2006): Els aiguamolls de l'Empordà. Paisatges d'aigua. Publicacions de l'Abadia de Montserrat. Barcelona.

SECRETARÍA GENERAL DE TURISMO (2004): El turismo de naturaleza en España y su plan de impulso. Ministerio de Industria, Turismo y Comercio. Madrid.

SUCH, M.P. (2003): «Ordenación del uso público de un espacio natural protegido en un destino turístico de masas: las lagunas de Torrevieja y La Mata», en Investigaciones Geográficas, ${ }^{\circ}$ 30, pp. 47-75.

TAPPER, R. (2006): Wildlife watching and tourism. A study on the benefits and risks of a fast growing tourism activity and its impacts on species. UNEP / CMS Secretariat. Bonn.

TAYLOR, D. (2004): Managing the visitor experience within Europe's protected areas. PAN Parks Foundation.

VERA, J.F. (coord.) (1997): Análisis territorial del turismo. Ed. Ariel. Barcelona. 
